University of Wollongong

Research Online

Faculty of Engineering and Information

Faculty of Engineering and Information

Sciences - Papers: Part A

Sciences

$1-1-2013$

\title{
Total electron-scattering cross sections from pyrimidine as measured using a magnetically confined experimental system
}

Martina C. Fuss

Consejo Superior De Investigaciones Científicas

Ana G. Sanz

Consejo Superior De Investigaciones Científicas

Francisco Blanco

Universidad Complutense, Avda. Complutense Sn, Madrid, Spain

Juan Carlos Oller

CIEMAT

Paulo Limao-Vieira

Universidade Nova De Lisboa

See next page for additional authors

Follow this and additional works at: https://ro.uow.edu.au/eispapers

Part of the Engineering Commons, and the Science and Technology Studies Commons

Research Online is the open access institutional repository for the University of Wollongong. For further information contact the UOW Library: research-pubs@uow.edu.au 


\title{
Total electron-scattering cross sections from pyrimidine as measured using a magnetically confined experimental system
}

\author{
Abstract \\ In this paper, an apparatus for measuring electron-scattering cross sections while applying a strong axial \\ magnetic field is described. The first original experiment performed with this setup is the measurement of \\ the total cross sections for pyrimidine (1,3-diazine), which are presented for the incident electron energy \\ range of 8-500 eV. Generally good agreement with earlier theoretical predictions is found after accounting \\ for the angular acceptance (angular resolution for forward scattering) of the apparatus.

\section{Keywords} \\ cross, scattering, electron, total, sections, measured, confined, pyrimidine, magnetically, experimental, \\ system

\section{Disciplines \\ Engineering | Science and Technology Studies}

\section{Publication Details} \\ Fuss, M. C., Sanz, A. G., Blanco, F., Oller, J. Carlos., Limao-Vieira, P., Brunger, M. J. and Garcia, G. (2013). \\ Total electron-scattering cross sections from pyrimidine as measured using a magnetically confined \\ experimental system. Physical Review A - Atomic, Molecular, and Optical Physics, 88 (4), \\ 042702-1-042702-8.
}

\section{Authors}

Martina C. Fuss, Ana G. Sanz, Francisco Blanco, Juan Carlos Oller, Paulo Limao-Vieira, Michael J. Brunger, and Gustavo Garcia 


\title{
Total electron-scattering cross sections from pyrimidine as measured using a magnetically confined experimental system
}

\author{
Martina C. Fuss, ${ }^{1}$ Ana G. Sanz, ${ }^{1}$ Francisco Blanco, ${ }^{2}$ Juan Carlos Oller, ${ }^{3}$ Paulo Limão-Vieira, ${ }^{4}$ \\ Michael J. Brunger, ${ }^{5,6}$ and Gustavo García ${ }^{1,7, *}$ \\ ${ }^{1}$ Instituto de Física Fundamental, Consejo Superior de Investigaciones Científicas, 28006 Madrid, Spain \\ ${ }^{2}$ Departamento de Física Atómica, Molecular y Nuclear, Universidad Complutense de Madrid, 28040 Madrid, Spain \\ ${ }^{3}$ Centro de Investigaciones Energéticas, Medioambientales y Tecnológicas (CIEMAT), 28040 Madrid, Spain \\ ${ }^{4}$ Laboratório de Colisões Atómicas e Moleculares, CEFITEC, Departamento de Física, Faculdade de Ciências e Tecnologia, \\ Universidade Nova de Lisboa, 2829-516 Caparica, Portugal \\ ${ }^{5}$ ARC Centre for Antimatter-Matter Studies, School of Chemical and Physical Sciences, Flinders University, G.P.O. Box 2100, \\ Adelaide, South Australia 5001, Australia \\ ${ }^{6}$ Institute of Mathematical Sciences, University of Malaya, Kuala Lumpur, Malaysia \\ ${ }^{7}$ Centre for Medical Radiation Physics, University of Wollongong, New South Wales 2522, Australia
}

(Received 11 June 2013; published 7 October 2013)

\begin{abstract}
In this paper, an apparatus for measuring electron-scattering cross sections while applying a strong axial magnetic field is described. The first original experiment performed with this setup is the measurement of the total cross sections for pyrimidine (1,3-diazine), which are presented for the incident electron energy range of 8-500 eV. Generally good agreement with earlier theoretical predictions is found after accounting for the angular acceptance (angular resolution for forward scattering) of the apparatus.
\end{abstract}

DOI: 10.1103/PhysRevA.88.042702

PACS number(s): 34.80.-i, 34.90.+q

\section{INTRODUCTION}

It is well known [1] that high-energy radiation produces abundant secondary electrons $\left(\sim 4 \times 10^{4}\right.$ per $\mathrm{MeV}$ of energy primarily transferred), which are the main source of the energy deposition map and radiation damage in biological tissues. These low-energy, possibly even subionizing, electrons play an important role in inducing damage such as strand breaks or molecular dissociations in biomolecular systems, as has been extensively demonstrated [e.g. 2-4]. Therefore, when studying radiation effects in biological media, it is essential that the particular electron interaction parameters (integral and differential cross sections, energy loss spectra, and partial scattering cross sections especially for dissociative interactions or radical generation) in the whole energy range are well known.

In view of this, we aim at contributing to the electronmolecule-scattering data pool with experiments carried out using a newly constructed apparatus that we describe later in this paper. This system for measuring electron-scattering cross sections (CSs) is based on the strong axial magnetic confinement of the electrons inside the collision chamber and utilizes their energy analysis and detection. The technique permits, in principle, the simultaneous measurement of the total scattering CS, integral cross sections for elastic and different inelastic processes (depending on the associated energy loss), and absolute differential CSs by varying the relative intensity of the magnetic field in the collision and detection region [5]. The first molecular target studied is pyrimidine, whose total scattering CS we have measured for incident energies in the range $8-500 \mathrm{eV}$.

\footnotetext{
*g.garcia@iff.csic.es
}

Pyrimidine $\left(\mathrm{C}_{4} \mathrm{H}_{4} \mathrm{~N}_{2}, 1,3\right.$-diazine $)$ forms the molecular basis of several biological molecules, such as the DNA and RNA bases cytosine, thymine, and uracil, and also thiamine and alloxan. In contrast to those potentially more interesting species, pyrimidine is stable to heating and has its liquid phase at normal pressure close to room temperature, making it much more accessible for scattering experiments, particularly those carried out in the gaseous phase. It therefore constitutes a convenient model system for exploring electron-scattering properties of the previously mentioned biomolecules. Note that the prospect of building up the requisite data base for track simulations in matter, similar to what has been done for other organic molecules, e.g., Ref. [6], is of great interest and forms a major rationale behind this investigation.

Pyrimidine belongs to the $C_{2 v}$ point group and exhibits a strong permanent dipole moment of between 2.28 and 2.39 D [7-9], which leads us to expecting a certain prominence of rotational excitations $[10,11]$ in the scattering process at lower energies. We notice that in the last years, several electron-pyrimidine scattering studies have been published. In 2005, experiments on low-energy electron scattering from condensed pyrimidine were presented by Sanche's group [12]. Subsequently two experimental groups [13,14] published elastic differential cross sections (DCSs), presenting results for the combined energy range $3-300 \mathrm{eV}$. Jones et al. $[15,16]$ next measured differential electronic excitation CSs for various sets of unresolved states in the incident energy range $15-50 \mathrm{eV}$, while Hein et al. [17] studied dissociative excitation from threshold up to $375 \mathrm{eV}$. Furthermore, Linert et al. [18] have presented electron-impact ionization cross sections from threshold up to $150 \mathrm{eV}$. Electron energy loss spectra and the stopping power in pyrimidine $(20-3000 \mathrm{eV})$ have also been published recently [19]. An extensive study on the VUV electronic state spectroscopy of pyrimidine has been reported by Ferreira da Silva et al. [20] in the energy range 
3.7-10.8 eV together with a comprehensive electron energy loss spectroscopy (EELS) of the singlet excitations in the range 2-15 eV. However, experimental total scattering CSs have, to the best of our knowledge, only been obtained for positrons as a projectile $[10,21]$. This is something of an oversight by the scattering community, because the total CS, being the sum of the integral cross sections for all possible scattering processes, represents a vital self-consistency check for those integral cross sections.

Theoretical investigations on electron scattering from pyrimidine include our IAM-SCAR (independent atom model-screening-corrected additivity rule) calculations [10, $11,13]$ and $R$-matrix calculations by Mašín and Gorfinkiel [22], Mašín et al. [23], and Sanz et al. [11]. In addition, we also note the Schwinger multichannel calculations from Palihawadana et al. [14], and, most recently, scaled quasifree-scattering model calculations from Ferraz et al. [24].

The structure of the present paper is as follows. The next section gives a detailed description of the magnetically confined electron-scattering apparatus employed for the experiments in this study and explains its technical characteristics. Afterwards, in Sec. III, extra details regarding the experiments, such as the measurement protocols, the CS calculation, and the uncertainties are treated. The results obtained for total electron scattering from pyrimidine are then presented and compared to other available data in Sec. IV. Finally, some conclusions drawn from the present work are given together with some future perspectives.

\section{MAGNETICALLY CONFINED ELECTRON-SCATTERING SYSTEM}

An experimental system for measuring electron-scattering cross sections, within a strong axial magnetic field, has been constructed at the Instituto de Física Fundamental (CSIC) in Madrid. The functionality of this apparatus is based on the magnetic confinement of the electron beam from its entrance into the collision chamber, until its energy analysis and detection. In this way, scattered as well as unscattered electrons are guided in the forward direction while retaining all information about their energy and scattering angle, being detected together after the analysis of their energy. In principle, this makes it possible to undertake simultaneous absolute measurements of electrons scattered by (almost) all angles in a fashion similar to the system described in Ref. [5]. In this context, one should interpret the main magnetic field simply as a means of translating the electron-which exhibits the exact angle and energy that resulted from a potential collision-along the central axis to the end of the collision chamber. In the retarding potential analyzer (RPA) region, the beam confinement can be changed, and the independent magnetic field $B_{\mathrm{RPA}}$ (see below) can be used to select which electrons should be analyzed depending on the type of cross section to be measured. The present study centers on the total CS measurement described in detail in Sec. III.

\section{A. Design and technical characteristics}

A schematic diagram of the entire apparatus is given in Fig. 1(a). It consists of three regions (electron gun, collision

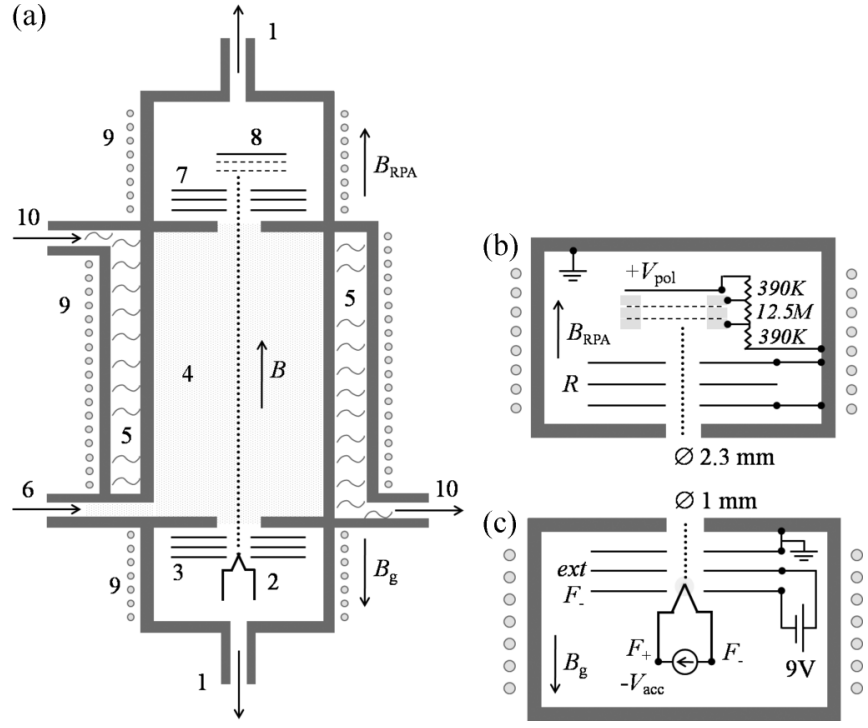

FIG. 1. (a) Schematic representation of the magnetically confined electron-scattering apparatus: 1-turbomolecular pumps, 2-electron-emitting filament, 3-extracting and accelerating electrodes, 4-collision chamber, 5-water jacket, 6-gas inlet, 7-retarding potential analyzer (RPA), 8-electron detector (microchannel plate assembly), 9—magnetic coils, 10—cooling liquid inlet or outlet. (b) and (c) Detailed view and electrical connections of the analyzer-detector region and the electron gun, respectively. (b) $R$-retarding electrode, $V_{\text {pol }}$ - polarization voltage of the detector assembly. (c) $V_{\text {acc }}$-accelerating voltage, $F_{+}$and $F_{-}$- positive and negative pins of the emitting filament, ext-extraction electrode.

chamber, and analyzer-detector region) which are connected by two small orifices $(1 \mathrm{~mm}$ and $2.3 \mathrm{~mm}$ in diameter). These regions are differentially pumped by means of two turbomolecular pumps of throughput $450 \mathrm{l} / \mathrm{s}$ (electron gun region; Leybold Heraeus, Germany) and 70 1/s (analyzerdetector region; Varian, Italy). They reach a background pressure of the order of $10^{-7} \mathrm{mbar}$, which can increase to $10^{-6}$ mbar during the measurements upon gas admission. All three compartments are surrounded by solenoids that produce the independent magnetic fields $B_{\mathrm{g}}, B$, and $B_{\mathrm{RPA}}$, respectively. These are used to magnetically confine all the electrons upon entering the collision chamber and, after a potential scattering event, guide them to the retarding potential analyzer while retaining all the information about their energy and scattering angle. Electrons scattered backwards (by angles $>90^{\circ}$ ) are reflected at the chamber entrance and traverse the collision region once again before being analyzed.

The electron gun [Fig. 1(c)] is formed by a commercial tungsten filament (Agar Scientific, United Kingdom) and three electrodes. The diameter of the first aperture, which is penetrated by the filament tip, is $2.5 \mathrm{~mm}$ while the remaining two apertures have $1 \mathrm{~mm}$ diameters. Electrons are obtained by thermionic emission from the filament which is held at a negative bias $-V_{\text {acc}}$. The primary beam is generated by an extraction potential $V_{\text {ext }}$, then accelerated by a grounded electrode. This beam finally enters the collision chamber through a $1 \mathrm{~mm}$ orifice. Note that the magnetic field $B_{\mathrm{g}}$ of the electron gun region is oriented to oppose the main field $B$ of the collision chamber, in order to compensate for any peripheral 
components of $B$ inside the electron gun. This configuration ensures a low angular spread of the primary electron beam, since those electrons leaving the filament in directions other than the chamber central axis are not magnetically guided through the collimators.

The cylindrical collision chamber itself has a geometrical length of $140 \mathrm{~mm}$ and an inner diameter of approximately $60 \mathrm{~mm}$. Both are sufficiently large, compared to the collision chamber end apertures, to guarantee a well-defined region of constant pressure inside this chamber and have steep pressure gradients at both its ends. The surrounding magnetic coils (3.1 mm thick copper wire insulated with special $200^{\circ} \mathrm{C}$ heat-resistant enamel) produce a maximum magnetic field $B$ of about $0.2 \mathrm{~T}$ when operated with a current of $35 \mathrm{~A}$. The target species is introduced into the system via a variable leak valve (Agilent Technologies, California). The pressure in the chamber is determined by a Baratron capacitance manometer (MKS, Germany) and the temperature at the inner wall of the collision chamber (this through good thermal contact is supposed to accurately reflect the gas temperature) is measured using a K-type thermocouple.

After passing into the detector region, electrons are selected by a retarding potential analyzer. This RPA consists of three electrodes of $2.3 \mathrm{~mm}$ aperture diameter, separated from each other by $2 \mathrm{~mm}$, with each biased according to ground/ $-V_{R} /$ ground [see the electrical scheme in Fig. 1(b)]. Therefore, only those electrons with parallel (axial) components of the kinetic energy $\geqslant e V_{R}$, where $e$ represents the elementary charge, can continue towards the detector. The other electrons which do not meet this criterion are rejected by the potential barrier and trapped in the chamber. After the analyzer, the detector assembly is located. It is formed by two microchannel plates (Hamamatsu Photonics, Japan), sandwiched between three annular electrodes, followed by the anode as depicted in Fig. 1(b). The typical polarization voltage $V_{\text {pol }}$ applied to the anode is $\sim 2 \mathrm{keV}$, resulting in about $940 \mathrm{~V}$ across each microchannel plate (MCP).

The electron detector is operated in a single-pulse counting mode. The electron cascades (corresponding to single electrons) generated in the MCPs are accelerated to the anode causing a negative current. This current now enters a custom-built charge-sensitive preamplifier which outputs voltage pulses of $\geqslant 10 \mathrm{meV}$ height. These are next amplified by a Canberra (Connecticut) amplifier, and the signal obtained is passed on to a constant fraction discriminator (Ortec, Tennessee) for eliminating any electronic and detector noise. The resulting standard rectangular pulses are transferred to a data acquisition system (National Instruments, Texas) connected to a PC running a custom LabView (National Instruments) program which finally counts the pulses.

\section{B. Focusing and energy resolution}

The electron beam enters the collision chamber after collimation through a $1 \mathrm{~mm}$ diameter orifice. Inside the chamber the high magnetic field $B$, up to a maximum of $\sim 0.2 \mathrm{~T}$, is capable of maintaining a similar beam diameter after scattering for electron energies up to $2 \mathrm{keV}$. Indeed the maximum gyroradius of the electron's perpendicular circular (a)

(b)

FIG. 2. Cross section of the electron beam after traversing the collision chamber, as visualized with a $\mathrm{ZnS}$ luminescence detector and being photographed. The blue color channel of the image files was inverted in order to obtain the present figure. (a) $2 \mathrm{keV}$ electrons in vacuum. (b) $2 \mathrm{keV}$ electron beam in 20 mTorr of $\mathrm{SF}_{6}$. In both cases, the beam diameter is $<1 \mathrm{~mm}$.

motion-this occurs after elastic scattering by $90^{\circ}$ at the highest incident energy - is calculated to be $0.5 \mathrm{~mm}$.

Beam focus and alignment in vacuum, and subsequently in a $\mathrm{SF}_{6}$ gas-filled chamber, have been verified to comply with these requirements (see Fig. 2) and do not exceed $1 \mathrm{~mm}$ diameter and $0.5 \mathrm{~mm}$ lateral displacement from the geometrical axis of the chamber (this is due to the mechanical tolerances of the collimators as well as the filament and its base). The effective localization of electrons after scattering, and before entering the analyzer, is thus expected to be within a radius of $\leqslant 1 \mathrm{~mm}$ around the axis for all incident energies up to $2 \mathrm{keV}$.

The energy resolution $\delta E$ of the electron beam in a given configuration (i.e., a given incident energy, magnetic fields applied, and filament current and emission rate) is obtained from the transmission curve $I\left(V_{R}\right)$ in vacuum, where $I$ is the transmitted beam intensity (electron count rate) and $V_{R}$ is the retarding potential. It is acquired by ramping the retarding potential via our LabView electron counting program, so that a sharp decrease in intensity appears near the beam energy. An example for $E=70 \mathrm{eV}$, including the energy distribution density (Gaussian function fitted to the derivative of the transmission curve), is depicted in Fig. 3. In this study, energy resolution is defined as $\delta E=e\left(V_{R, 90}-V_{R, 10}\right) / 2$, with $V_{R, 90}$ and $V_{R, 10}$ being the retarding potentials leading to $90 \%$ and $10 \%$ of transmitted electrons. This was generally found to be similar to the FWHM (full width at half maximum) from the derivatives of transmission curves and yielded typical values $\leqslant 1.3 \mathrm{eV}$ (with a best resolution of $0.25 \mathrm{eV}$ ). Note also that the angular spread of the primary beam crucially influences the cutoff behavior of the transmission curve.

When a target species is present in the collision chamber, the transmission curve adopts a very different profile. In particular it no longer possesses a sharp beam cutoff (see Fig. 3), because the energy corresponding to the parallel motion component after scattering $\left(E_{\|}^{\prime}\right)$ in that case responds to changes both in the electron energy $E$ and scattering angle $\theta \quad\left(E_{\|}^{\prime}=E^{\prime} \cos ^{2} \theta\right.$, where $E^{\prime}$ is the electron energy after collision). As a consequence, the curve $I(R)$ shows undulations that result from a superposition of the energy loss spectrum and the angular distribution of the scattered electrons. 


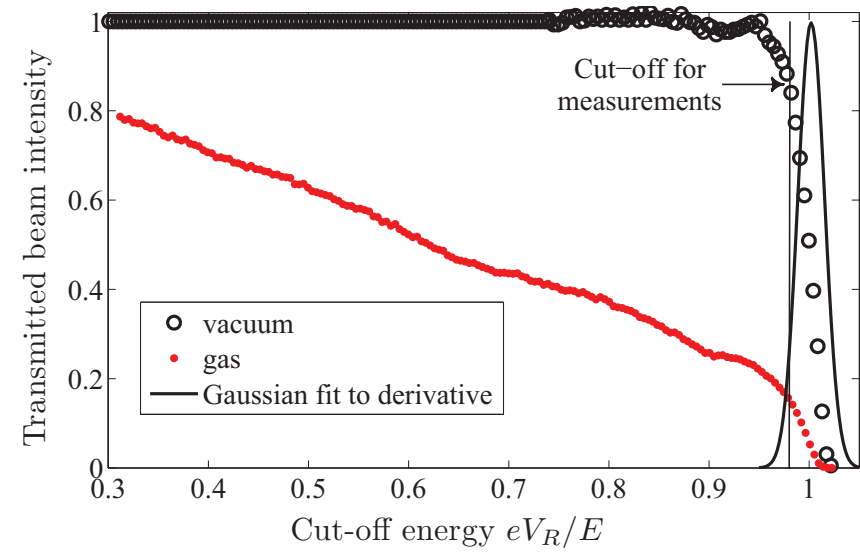

FIG. 3. (Color online) Typical example of a transmission curve in vacuum and also in 1.5 mTorr of pyrimidine ("gas") at an incident energy $E$ of $70 \mathrm{eV}$. Also plotted is a Gaussian function fitted to the derivative of the vacuum transmission curve (electron energy distribution density), scaled to a maximum of 1 . The transmitted beam intensity is normalized to the maximum $\left(V_{R}=0\right)$. The cutoff energy of the retarding potential analyzer is given relative to the incident energy $E$.

\section{Temperature control}

To dissipate heat from the main magnetic coils and from the Leybold pump, a liquid cooling circuit was installed. It consists of two parallel cooling loops, each equipped with a variable speed (max. 1200 l/h) water pump (Swiftech, California) leading to the solenoid and the vacuum pump. Those branches join before reaching an air-cooled radiator (Aquacomputer, Germany), where the fluid is ventilated by nine $12 \times 12 \mathrm{~cm}^{2}$, $1850 \mathrm{rpm}$ fans. Distilled water with approximately $5 \%$ of an antifungal coolant (Swiftech) is used as the cooling liquid. In addition to the water cooling, the solenoid can be externally air-cooled by a number of different fans. This configuration ensures that the turbomolecular pump is reliably cooled in a constant manner, while the temperature of the collision chamber beneath the main solenoid can also be controlled to some degree in order to avoid excessive condensation on the chamber wall (the adjustable chamber cooling parameterspump speed of the "magnetic coil" branch of the configuration, use of the additional fans - are flexibly adapted to the required chamber temperature).

Solid samples or volatile but "sticky" liquids, which have their melting point close to room temperature, such as pyrimidine, can be introduced into the present apparatus thanks to local heating of the inlet valve. A total of $69.6 \mathrm{~cm}^{2}$ of etched foil silicone heater mats (RS-Amidata, United Kingdom) are attached to the inlet valve and the sample container (a $\mathrm{CF}$ 16-40 adapter used as a steel flask). Powered with $28 \mathrm{~W}$, the flask and valve reach a temperature of around $40{ }^{\circ} \mathrm{C}$; although higher temperatures are also attainable.

\section{MEASUREMENT PROTOCOLS}

Pyrimidine (1,3-diazine), with a stated purity of 99\%, was purchased from Sigma-Aldrich and further purified through the performance of some freeze-pump-thaw cycles. The preliminary tests and validation experiments that we carried out revealed certain critical aspects of the experimental work flow regarding the reproducibility and accuracy of the total cross sections obtained using the present apparatus. Those protocols were all taken into account during the preparation, measurement, and data analysis carried out in this study and can be summarized as follows: (i) The temperature of the solenoids and of the emitting filament need to be stabilized before starting a set of measurements. Temperature changes during our measurements are within $\pm 1^{\circ}$. (ii) Signal losses due to the rate-dependent detector dead time have to be strictly avoided, since within an attenuation curve they would depend on the gas pressure and would skew that curve. (iii) The cutoff behavior and resolution of the transmission curve have to be verified by an adequate adjustment of the magnetic fields before introducing the sample into the collision chamber. Whereas the main role of $B_{\mathrm{g}}$ is to counteract $B$ in the electron gun region and so reduce the angular spread, $B_{\mathrm{RPA}}$ in the present experiments is used to help focus the electrons during their passage through the analyzer. (iv) To improve the effectiveness of the energy analyzer during the attenuation measurements, the selected retarding potential cutoff value transmits only the more energetic $85 \%$ of the initial beam intensity in vacuum (see Fig. 3). (v) To minimize any gas effects (enhancement) in the filament emission, the filament is permitted to stabilize before any measurements are performed. (vi) Finally, any measurement cycles presenting an apparent hysteresis between pressure increase and reduction, or curves that deviate from an exponential function [see Eq. (1)], are excluded from further analysis.

A series of measurements yielding the total CS value at a given incident electron energy typically consists of 7-10 attenuation curves acquired in alternating directions (increasing or decreasing pressure) with each comprising normally 7-12 data pairs (pressure $p$ and intensity $I$ ). Representative examples at different incident energies can be seen in Fig. 4. Due to the occasional observation of plasma focusing effects around the chamber apertures at very low ( $\leqslant 1$ mTorr) sample pressures, those pressures are avoided where possible during the current measurements. Data points were fitted by an exponential

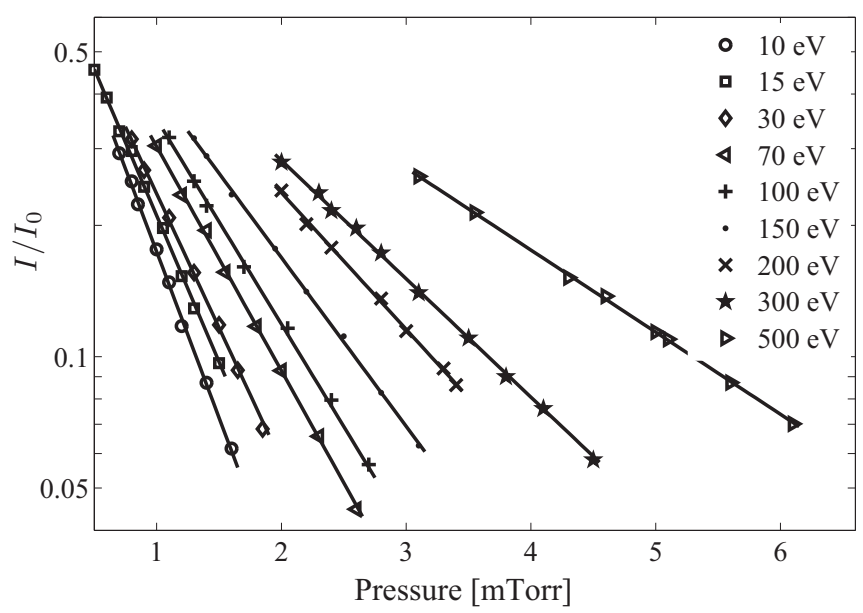

FIG. 4. Example of representative attenuation curves for incident electron energies from 10 to $500 \mathrm{eV}$ [original data points $(p, I)$ and exponential fit function] for scattering from pyrimidine. See legend in figure for further details. 
attenuation curve $I(p)$, from which the total scattering cross section $\sigma_{\text {expt }}$ is obtained according to the Beer-Lambert law:

$$
I=I_{0} e^{-n l \sigma_{\mathrm{expt}}}=I_{0} e^{-p l \sigma_{\mathrm{expt}} / k T} .
$$

Here, $I_{0}$ is the intensity of the unattenuated beam (in vacuum), $n$ is the number density of the target gas, $l=141.3 \mathrm{~mm}$ is the effective collision chamber length (see Sec. III A), $k$ is the Boltzmann constant, and $T=\sqrt{T_{\mathrm{c}} T_{\mathrm{m}}}$ is the gas temperature (K) calculated according to the thermal transpiration effect [25] between the manometer at $T_{\mathrm{m}}$ and the collision chamber at $T_{\mathrm{c}}$.

To validate and benchmark our apparatus and our measurement procedures, we conducted preliminary total electronscattering cross section measurements with argon at some incident energies in the range 20-500 eV. Excellent accord ( $\leqslant 2.5 \%$ difference) was found between our total CS results and those published by Nickel et al. [26], Szmytkowski et al. [27], and Zecca et al. [28].

\section{A. Statistical uncertainties and angular acceptance}

Experimental uncertainties on the total CS measurements carried out with the magnetically confined electron-scattering system are introduced by different elements. The effective collision chamber length $l=l_{\text {geom }}+d_{1}+d_{2}$ (where $l_{\text {geom }}$ is the geometrical length and $d_{1}$ and $d_{2}$ are the diameters of the scattering cell entrance and exit aperture, where the pressure falls off) is afflicted with an uncertainty of $\pm 1.1 \%$. Similarly pressure measurements inside the chamber are accurate to $<0.5 \%$, according to the manufacturer's calibration. The collision chamber temperature is determined with an uncertainty of $1 \%$, by the K-type thermocouple, in the range of temperatures observed. In addition the incident beam energy is affected by an uncertainty of approximately $+1 \mathrm{eV}$, when using as an estimate the difference of the acceleration potential (at the filament) and the beam cutoff energy (at the analyzer). This corresponds to $0.2-10 \%$ of the incident energy, in the energy range of this study, and converts to an uncertainty between $0.2 \%$ and $2 \%$ on the resulting total CS value (at 500 and $10 \mathrm{eV}$, respectively). The standard deviation among the measurements of each series, calculated as a measure of the experimental reproducibility, was typically $\leqslant 4 \%$. This amount comprises the factors of filament emission stability, temperature stability, and signal fluctuations caused by the electronic circuitry, as well as the uncertainty in the determination of the attenuation curve fit function (exponent) from the measured values. Combining the aforementioned factors, one obtains a general precision on the present experimental total cross section determination of $\sim 4.4 \%$ at incident energies $\geqslant 20 \mathrm{eV}$, and of $<5 \%$ for incident energies $\leqslant 15 \mathrm{eV}$.

In addition to the general uncertainty of the statistical nature of our measurements, as discussed above, the angular acceptance $\delta \theta$ of the apparatus is also a limiting aspect and presents an important source of systematic error. The strong magnetic confinement used in this experiment converts any angular deflection due to scattering processes into a decrement of the axial component of the velocity vector $\left(v_{\|}^{\prime}\right)$ which turns into a loss of the kinetic energy after scattering in the axial (forward) direction $\left(E_{\|}^{\prime}\right)$. Hence, there are some angular regions $(0 \leqslant \theta \leqslant \delta \theta$ and $180-\delta \theta \leqslant \theta \leqslant 180)$, limited by the energy resolution $(\delta E)$, in which elastically scattered electrons are accounted for as "unscattered" by our analyzer-detector system, therefore leading to an observed cross-section value lower than the "true" one. A similar argument can be used for rotationally inelastically scattered electrons, since the excitation energy related to these processes is much lower than the energy resolution of this experimental system. Indeed, depending on the energy resolution attained, a contribution from vibrational excitation scattered electrons is also in principle possible. The relations between the angular acceptance and energy resolution limits are given by the following expressions:

$$
\begin{gathered}
E_{\|}^{\prime}=E \cos ^{2}(\delta \theta)=E-\delta E, \\
\delta \theta=\arccos \sqrt{1-\frac{\delta E}{E}} .
\end{gathered}
$$

On the contrary, electronically inelastically scattered electrons are efficiently discriminated by the retarding potential analyzer. Consequentially, any comparison to other total CS data needs to take into account that the present experimental values $\left(\sigma_{\text {expt }}\right)$ represent, in fact, a partial value:

$$
\begin{aligned}
\sigma_{\text {expt }}(E) \approx & \sigma(E)-\sigma_{\text {forw }}(E)<\sigma(E) \text { with } \\
\sigma_{\text {forw }}= & 2 \pi\left(\int_{0}^{\delta \theta} \frac{d\left(\sigma_{\mathrm{el}}+\sigma_{\text {rot }}\right)}{d \Omega} \sin \theta d \theta\right. \\
& \left.+\int_{180-\delta \theta}^{180} \frac{d\left(\sigma_{\mathrm{el}}+\sigma_{\text {rot }}\right)}{d \Omega} \sin \theta d \theta\right),
\end{aligned}
$$

where $\sigma_{\mathrm{el}}$ and $\sigma_{\text {rot }}$ denote the integral elastic and rotational excitation CS, respectively. Although the contribution of the vibrational excitation of the ground state to $\mathrm{z}(5)$ should be also considered, it represents less than $1 \%$ of that of the elastic scattering, in terms of cross sections [12,14]. We have thus neglected the respective contribution to the systematic error in comparison to that derived for the elastic scattering and rotational excitations.

For the measurements we carried out, the angular acceptance $\delta \theta$ lies in the range $3.3^{\circ}-14.6^{\circ}$ and is explicitly stated for each experimental CS value in Sec. IV. An estimation of the systematic error can be derived from the results of our IAM-SCARD (IAM-SCAR including dipole interactions) calculations [10,14] (see Table I).

\section{RESULTS}

The experimental cross sections $\sigma_{\text {expt }}(E)$ we obtained in the incident energy range $8-500 \mathrm{eV}$ are presented in Table I. Also included are the angular acceptance of each measurement and an estimate of the systematic error $\delta \sigma_{\text {expt }}(E)$, calculated as

$$
\delta \sigma_{\text {expt }}(E)=\frac{\sigma_{\text {forw }}(E)}{\sigma(E)-\sigma_{\text {forw }}(E)} \sigma_{\text {expt }}(E) .
$$

For this estimation, our IAM-SCARD calculation, including rotational contributions treated as a dipole interaction, [10,11] is used as a reference.

Figure 5 depicts the present experimental results together with our previously published IAM-SCAR(D) results [10,11], the recent scaled quasi-free-scattering model (SQFSM) total CS calculation of Ferraz et al. [24], the low-energy $R$ matrix calculations of Sanz et al. [11], and experimental 
TABLE I. Present total electron-scattering CSs, $\sigma_{\text {expt }}(E)$, from pyrimidine (1,3-diazine) in units of $10^{-20} \mathrm{~m}^{2}$. The reproducibility (standard deviation among measurements for the same incident energy, see Sec. III A) in our data is given as the percentage of the experimental value. The relevant energy resolution $\delta E$, angular acceptance $\delta \theta$, and the corresponding theoretical values $\sigma-\sigma_{\text {forw }}$ are also given for each measurement. The estimation of the systematic error $\left(\delta \sigma_{\text {expt }}\right)$ due to the apparatus' angular acceptance, is calculated using our IAM-SCARD theoretical results and is stated as a percentage of the experimental value.

\begin{tabular}{lcccccc}
\hline \hline $\begin{array}{l}\text { Energy } \\
(\mathrm{eV})\end{array}$ & $\sigma_{\text {expt }}$ & Reproduc. & $\delta E(\mathrm{eV})$ & $\delta \theta(\mathrm{deg})$ & $\sigma-\sigma_{\text {forw }}$ & $\delta \sigma_{\text {expt }}$ \\
\hline 8.0 & 41.1 & 4.0 & 0.51 & 14.6 & & 61.4 \\
10 & 38.9 & 1.7 & 0.63 & 14.5 & 45.0 & 56.7 \\
12 & 36.9 & 2.5 & 0.37 & 10.1 & & 45.4 \\
15 & 35.4 & 7.8 & 0.50 & 10.5 & 41.7 & 42.3 \\
20 & 34.5 & 4.4 & 0.51 & 9.2 & 39.7 & 34.8 \\
25 & 33.4 & 1.5 & 0.86 & 10.7 & & 34.1 \\
30 & 32.4 & 6.8 & 0.42 & 6.8 & 37.7 & 24.2 \\
40 & 29.2 & 1.7 & 1.2 & 10.1 & 33.3 & 27.2 \\
50 & 29.3 & 3.9 & 0.70 & 6.8 & 32.3 & 19.6 \\
60 & 28.0 & 4.8 & 0.59 & 5.7 & & 16.1 \\
70 & 26.9 & 1.8 & 1.3 & 7.9 & 28.5 & 18.8 \\
100 & 24.0 & 1.4 & 1.4 & 6.9 & 24.6 & 20.8 \\
150 & 20.3 & 3.1 & 1.3 & 5.4 & 21.4 & 12.6 \\
200 & 15.8 & 1.8 & 4.7 & 8.8 & 16.8 & 23.9 \\
300 & 14.6 & 1.8 & 1.0 & 3.3 & 15.0 & 10.6 \\
400 & 11.6 & 1.4 & 4.2 & 5.9 & 11.5 & 20.8 \\
500 & 9.69 & 1.9 & 5.4 & 6.0 & 9.84 & 23.0 \\
\hline \hline
\end{tabular}

integral cross-section data from Palihawadana et al. [14] on elastic scattering. No other electron total CSs were found for comparison in the literature. Note that, due to some very minor

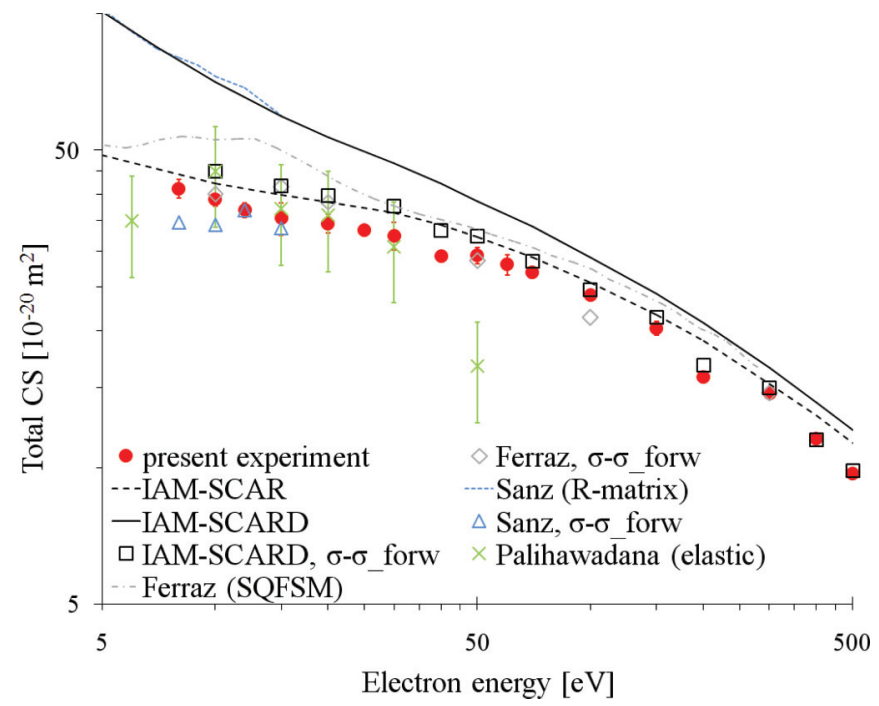

FIG. 5. (Color online) The total CS for electron scattering from pyrimidine. The error bars denoted for the present experiment $(\bullet)$ reflect the combined statistical uncertainty of a data point (see Sec. III A). CSs $\sigma-\sigma_{\text {forw }}$, emulating the experimental conditions, are given for the theoretical IAM-SCARD and Born-dipole-corrected SQFSM [24] and $R$-matrix [11] results in addition to the exact values. Integral elastic CSs from Palihawadana et al. [14] are also depicted for comparison. geometrical misalignment of the focusing elements in the electron gun, our energy and consequently angular resolutions presented a sudden increase in value for three of the high incident energies-200, 400, and $500 \mathrm{eV}$ as can be seen in Table I-which results in relatively lower total CS values for those energies. To account for the angular acceptance $\delta \theta$ of the apparatus observed during each measurement, the present experimental values should actually be compared to $\sigma-\sigma_{\text {forw }}$, the theoretical predictions of $\sigma$ under the experimental conditions, which are also included for all of the theoretical total CSs mentioned.

It can be seen from Fig. 5 that the experimental values generally exhibit good agreement $(<5-10 \%$ difference) with the IAM-SCARD scattering cross section calculated as in experimental conditions, $\sigma-\sigma_{\text {forw }}$, although higher differences (up to 16\%) appear in the energy range $8-40 \mathrm{eV}$, where the experimental values lie below theory. Both sets of results, however, agree within the combined uncertainty limits. When comparing the present experiment to the SQFSM results $\sigma-\sigma_{\text {forw }}$ derived from Ref. [24], a similar overall level of agreement is observed, although those $\sigma-\sigma_{\text {forw }}$ for some energies tend to be lower than the IAM-SCARD results, in analogy with the original (full) CS values. The differences in the total CSs, in the overlapping energy range, with the $R$-matrix values $\sigma-\sigma_{\text {forw }}$ derived from Sanz et al. [11] fall also within a very similar magnitude (0.1-17\%). However in this case the theoretical prediction consistently underestimates the measured CS. These discrepancies between our total CS and the different calculations (especially in regard to the IAM-SCARD and $R$-matrix, which are closely agreeing in the total CS), when comparing $\sigma-\sigma_{\text {forw }}$, clearly indicate the importance of the elastic angular distribution when attempting to compare experimental and calculated total CS values. It is our premise that a valid comparison can only be made after accounting for the known experimental limitations. Nonetheless, the differences found in all our comparisons are still quite reasonable, taking into account that the calculated dipole- or Born-corrected elastic DCSs all present large gradients in the forward direction that, together with the precision of the angular acceptance determination, limit the accuracy of the partial integration necessary for computing $\sigma-\sigma_{\text {forw }}$. Additionally, the theoretical absorption CSs from Refs. [10,24] only start to rise for the lower energies studied here and might thus be affected by a higher uncertainty in the sense of an underestimation in its true value. At the same time, the $R$-matrix approach does not account for ionization and so aims to provide an accurate total CS up to about $10 \mathrm{eV}$. Summarizing, a pleasing overall level of agreement between the present experimental total CS and previous theoretical studies $[11,14,24]$ is obtained, especially after confirming that the experimental data largely lie between the different theoretical results.

Comparison to the experimental integral elastic CSs of Ref. [14] seems less satisfactory at first sight, since those integral CSs are either very similar to or even higher than the present total CSs in the energy range $10-30 \mathrm{eV}$, in spite of the inelastic interactions that are expected there. This effect is partly attributed to the contrary nature of the systematic part of the experimental uncertainty in both cases. Namely, while in the total CS experiments, unresolved small-angle-scattering 


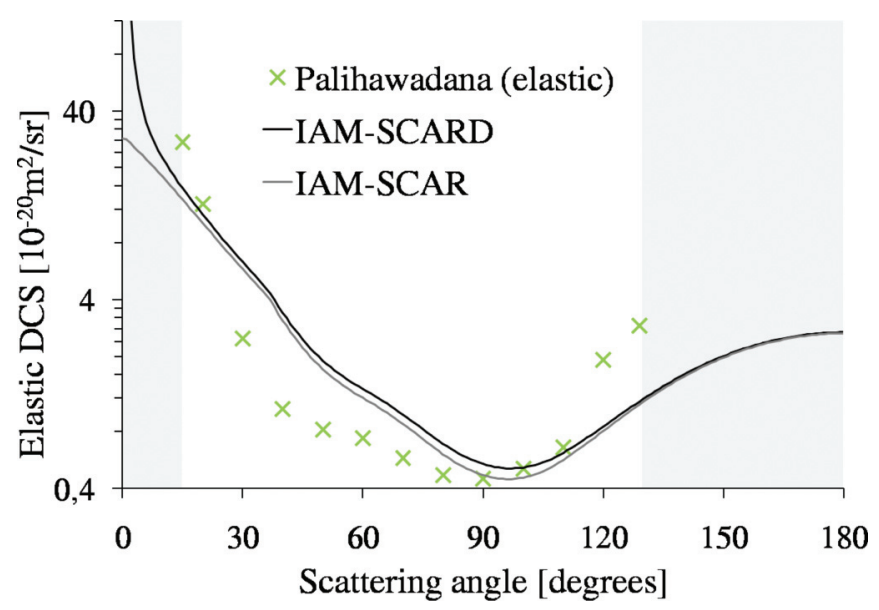

FIG. 6. (Color online) Example of an experimental elastic differential cross section [14] at $30 \mathrm{eV}$. The experimental uncertainty falls within the size of the symbol used. The gray background highlights the angular range not covered by the measurements and which has to be extrapolated prior to integration. The corresponding IAM-SCAR and IAM-SCARD (including rotational excitation) results are also shown.

tends to apparently decrease the measured CS, a very likely effect in the crossed-beam elastic scattering experiments, due to their energy resolution limitations, is the inclusion in the elastic CS of electrons that have in fact collided rotationally or vibrationally inelastically, leading to an overestimated "elastic" CS. At $50 \mathrm{eV}$, where both effects should be smallest within the overlapping energy range, a good agreement is found between the present $\sigma_{\text {expt }}\left(29.3 \times 10^{-20} \mathrm{~m}^{2}\right)$ and the sum of elastic integral CSs from Ref. [14] and ionization and electronic excitation CSs measured by Linert et al. [18] and Jones et al. [15], respectively $\left(26.1 \times 10^{-20} \mathrm{~m}^{2}\right)$, especially when taking into account that in Ref. [15], electronic states are included up to an excitation energy of $10 \mathrm{eV}$ and do thus represent rather a lower limit to the integral electronic excitation CS. Generally, it has to be kept in mind that the integral elastic CS derived in Ref. [14], as in most experimental studies on the elastic scattering CS, is actually the result of an extrapolation of the differential CS (DCS) towards $0^{\circ}$ and $180^{\circ}$ of the measurements conducted within a restricted angular range. As a consequence the elastic integral CSs are afflicted with considerably higher uncertainty (25\%) than the individual DCS data points (see Fig. 6 for an illustration). On these grounds no attempt was made from our side to obtain $\sigma-\sigma_{\text {forw }}$ from the original elastic DCS [14] for comparison since a considerable part of the angular range is actually missing from their measured DCS $\left(\theta \sim 15-130^{\circ}\right)$.

\section{CONCLUSIONS}

An experimental apparatus, making use of a strong axial magnetic field for studying electron scattering from molecules, has been constructed and its operation has been started with pyrimidine as the first target species. In view of the agreement between the measured total cross sections and comparisons with previous theoretical data, as discussed above, we conclude that the present system yields reliable values. This is particularly true when we allow for the experimental angular resolution in the forward direction (which determines the angular limit for distinguishing the scattered electrons) of the apparatus' configuration. In this respect our IAM-SCARD formalism has proven to be an extremely helpful complement to the experiment for providing CSs $\sigma-\sigma_{\text {forw }}$ emulating the exact experimental conditions so that a realistic comparison could be achieved. Certainly, the measurements present one step further towards the availability of a benchmarked selfconsistent pyrimidine data base for simulation [29] or other purposes. However, although the apparatus in its present form shows utility for total cross-section measurements, we aim to develop it further to achieve a better energy (and thus angular) resolution (or near constant resolution of approximately the lowest values obtained here, $0.4 \mathrm{eV}$ - this would translate to an angular resolution of $1.6^{\circ}-13^{\circ}$ for energies between 500 and $8 \mathrm{eV}$ ) before performing integral elastic, inelastic, or differential CS measurements. Given that the present apparatus features a flexible heating or cooling system that facilitates the use of solid (at room temperature) samples, the preference for future studies could lie in investigating interesting organic molecules with elevated melting points such as pyrazine, uracil, and the other DNA bases.

\section{ACKNOWLEDGMENTS}

This study was partially supported by the Ministerio de Ciencia e Innovación (FIS2009-10245 and FIS2012-31320) and the EU Framework Programme (COST Action MP1002). Technical support from the Spanish National Institute for Fusion Research of CIEMAT is acknowledged. M. C. F. acknowledges a PIF grant of the Comunidad Autónoma de Madrid. P. L. V. acknowledges FCT-MEC support through PEst-OE/FIS/UI0068/2011 and PTDC/FIS-ATO/1832/2012 grants. M. J. B. thanks the Australian Research Council for some financial support.
[1] I. Plante and F. A. Cucinotta, New J. Phys. 11, 063047 (2009).

[2] B. Boudaïfa, P. Cloutier, D. Hunting, M. A. Huels, and L. Sanche, Science 287, 1658 (2000).

[3] B. Boudaïffa, P. Cloutier, D. Hunting, M. A. Huels, and L. Sanche, Radiat. Res. 157, 227 (2002).

[4] M. A. Huels, B. Boudaïfa, P. Cloutier, D. Hunting, and L. Sanche, J. Am. Chem. Soc. 125, 4467 (2003).

[5] J. P. Sullivan, S. J. Gilbert, J. P. Marler, R. G. Greaves, S. J. Buckman, and C. M. Surko, Phys. Rev. A 66, 042708 (2002).
[6] M. Fuss, A. G. Sanz, A. Muñoz, T. P. T. Do, K. L. Nixon, M. J. Brunger, M.-J. Hubin-Franskin, J. C. Oller, F. Blanco, and G. García, Chem. Phys. Lett. 560, 22 (2013).

[7] G. L. Blackman, R. D. Brown, and F. R. Burden, J. Mol. Spectrosc. 35, 444 (1970).

[8] Z. Kisiel, L. Pszczolkowski, J. C. Lopez, J. L. Alonso, A. Maris, and W. Caminati, J. Mol. Spectrosc. 195, 332 (1999).

[9] P. Y. Chen and R. A. Holroyd, J. Chem. Phys. 100, 4491 (1996). 
[10] A. Zecca, L. Chiari, G. García, F. Blanco, E. Trainotti, and M. J. Brunger, J. Phys. B 43, 215204 (2010).

[11] A. G. Sanz, M. C. Fuss, F. Blanco, Z. Mašín, J. D. Gorfinkiel, F. Carelli, F. Sebastinelli, F. Gianturco, and G. García, Appl. Radiat. Isotop. (2013), doi:10.1016/j.apradiso.2013.01.031.

[12] P. L. Levesque, M. Michaud, and L. Sanche, J. Chem. Phys. 122, 094701 (2005)

[13] J. B. Maljković, A. R. Milosavljević, F. Blanco, D. Šević, G. García, and B. P. Marinković, Phys. Rev. A 79, 052706 (2009).

[14] P. Palihawadana, J. Sullivan, M. J. Brunger, C. Winstead, V. McKoy, G. García, F. Blanco, and S. J. Buckman, Phys. Rev. A 84, 062702 (2011).

[15] D. B. Jones, S. M. Bellm, F. Blanco, M. C. Fuss, G. García, P. Limão-Vieira, and M. J. Brunger, J. Chem. Phys. 137, 074304 (2012).

[16] D. B. Jones, S. M. Bellm, P. Limão-Vieira, and M. J. Brunger, Chem. Phys. Lett. 535, 30 (2012).

[17] J. D. Hein, H. Al-Khazraji, C. J. Tiessen, D. Lukic, J. A. Trocchi, and J. W. McConkey, J. Phys. B 46, 045202 (2013).

[18] I. Linert, M. Dampc, B. Mielewska, and M. Zubek, Eur. Phys. J. D 66, 20 (2012).

[19] R. Colmenares, A. G. Sanz, M. C. Fuss, F. Blanco, and G. García, Appl. Radiat. Isotop. (2013), doi:10.1016/j.apradiso. 2013.01.025.
[20] F. Ferreira da Silva, D. Almeida, G. Martins, A. R. Milosavljević, B. P. Marinković, S. V. Hoffmann, N. J. Mason, Y. Nunes, G. García, and P. Limão-Vieira, PhysChemChemPhys 12, 6717 (2010).

[21] P. Palihawadana, R. Boadle, L. Chiari, E. K. Anderson, J. R. Machacek, M. J. Brunger, S. J. Buckman, and J. P. Sullivan, Phys. Rev. A 88, 012717 (2013).

[22] Z. Mašín and J. D. Gorfinkiel, J. Chem. Phys. 137, 204312 (2012).

[23] Z. Mašín, J. D. Gorfinkiel, D. B. Jones, S. M. Bellm, and M. J. Brunger, J. Chem. Phys. 136, 144310 (2012).

[24] J. R. Ferraz, A. S. dos Santos, G. L. C. de Souza, A. I. Zaneleto, T. R. M. Alves, M.-T. Lee, L. M. Brescansin, R. R. Lucchese, and L. E. Machado, Phys. Rev. A 87, 032717 (2013).

[25] M. Knudsen, Ann. Physik 336, 205 (1909).

[26] J. C. Nickel, K. Imre, D. F. Register, and S. Trajmar, J. Phys. B 18, 125 (1985).

[27] C. Szmytkowski, K. Maciąg, and G. Karwasz, Phys. Scr. 54, 271 (1996).

[28] A. Zecca, S. Oss, G. Karwasz, R. Grisenty, and R. S. Brusa, J. Phys. B 20, 5157 (1987).

[29] M. C. Fuss, A. G. Sanz, A. Muñoz, F. Blanco, M. J. Brunger, S. J. Buckman, P. Limão-Vieira, and G. García, Appl. Radiat. Isotop. (2013), doi:10.1016/j.apradiso.2013.01.017. 